\title{
Micromachined 3D Millimeter-Wave and Terahertz Devices
}

\author{
Yi Wang ${ }^{*}$, Xiaobang Shang ${ }^{\dagger}$, Michael J. Lancaster ${ }^{\dagger}$, \\ "School of Electrical, Electronic and Computer Engineering \\ University of Greenwich (Medway Campus), ME4 4TB, Kent, UK \\ Email: yi.wang@gre.ac.uk \\ ${ }^{\dagger}$ School of Electrical, Electronic and Systems Engineering, University of Birmingham, B15 2TT, UK \\ Email: m.j.lancaster@bham.ac.uk, x.shang@bham.ac.uk
}

\begin{abstract}
Micromachining is a very promising technology to manufacture miniature three-dimensional (3D) devices at millimeter-wave (mm-wave) and terahertz ( $\mathrm{THz}$ ) frequencies. After a decade's development, this technology has begun to demonstrate its viability and capability. It has delivered devices with competitive performance to traditional metal machining or electroforming, for coaxial and waveguide structures with submillimeter dimensions. This paper will discuss three strands of work that tackle three main challenges - fabrications, designs and measurements - in this technology. Several passive devices will be presented to illustrate the progress made on the multilayered SU8 techniques. These include guided transmission structures and devices based on rectangular coaxial lines, waveguides and free-space frequency selective surfaces. The concerned frequency covers from $30 \mathrm{GHz}$ to $1 \mathrm{THz}$.
\end{abstract}

Index Terms - Micromachining, millimeter-wave devices, waveguides, frequency selective surfaces.

\section{INTRODUCTION}

As the frequency enters the millimeter-wave (mm-wave) and terahertz $(\mathrm{THz})$ region, the width of planar transmission lines gets so small that the power density becomes increasingly prohibitive to deliver decent transmission efficiency. To cope with this, three-dimensional (3D) transmission structures such as rectangular coaxial lines and waveguides are often resorted to. However, as their critical dimensions are down to the sub-millimeter regime at these frequencies, fabrication of such 3D structures becomes a significant challenge. For instance, the standard WR-03 waveguide $(220-325 \mathrm{GHz})$ has a cross-sectional aperture of $0.864 \mathrm{~mm} \times 0.432 \mathrm{~mm}$. A fabrication tolerance less than 10 $\mu \mathrm{m}$ and a surface roughness better than $100 \mathrm{~nm}$ are most desired. Although the high-precision metal machining or electroforming could meet the required fabrication accuracy, they tend to be very time-consuming and expensive. Their cost-effectiveness is poor. As the frequency increases and/or the internal structures get complicated, metal machining becomes impossible. The emergence of larger-scale applications of mm-wave and $\mathrm{THz}$ devices has stimulated the research into alternative fabrication techniques for $3 \mathrm{D}$ devices on the wafer level.

Various techniques have been investigated. Among them, UV-LIGA [1], PolyStrata ${ }^{\mathrm{TM}}$ [2], Silicon-DRIE [3] and thickresist photolithography [4], [5] are the most promising in providing the desired fabrication accuracy for up to $1 \mathrm{THz}$. LIGA is able to produce all-metal solid structures. PolyStrata demonstrated a high level of integration on the wafer. Compared with these, silicon-DRIE is a more accessible technique. As a competing technique with DRIE, the thickresist technology offers better cost-effectiveness for its lower facility requirement while producing the best surface smoothness on the vertical walls. Interestingly, some of these developments coincide with the rapid adaption of the concept of additive manufacturing. This paper focuses on the multilayer fabrication technique using the thick photoresist SU8.

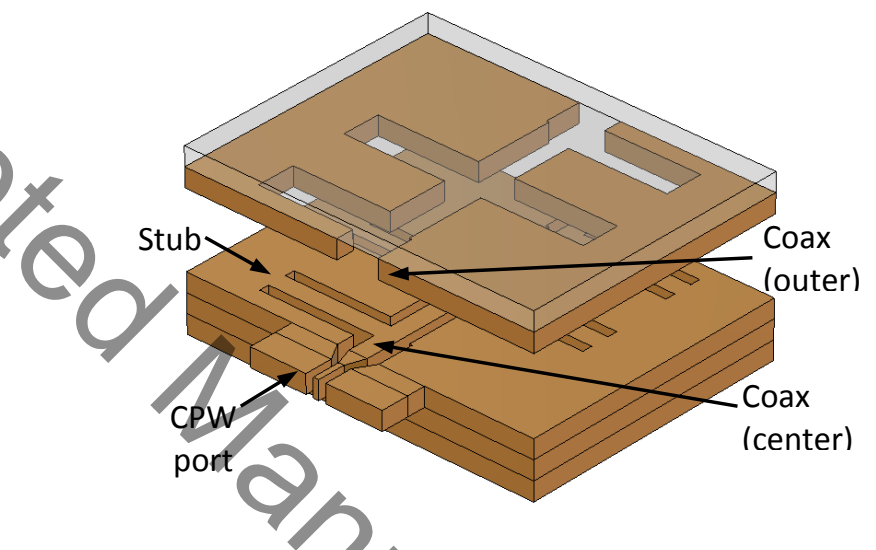

Fig.1. A rectangular coaxial line filter formed of five equal-thickness layers (for viewing the top two layers are separated and the top layer is made transparent).

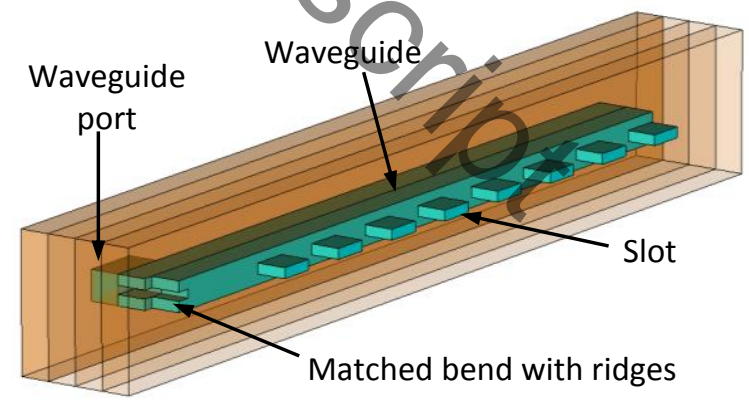

Fig. 2. A rectangular-waveguide antenna formed of four equalthickness layers. (The outstanding structure in blue in the middle represents the air-filled channel and slots, surrounded by conductors set to be transparent in the graph.) 


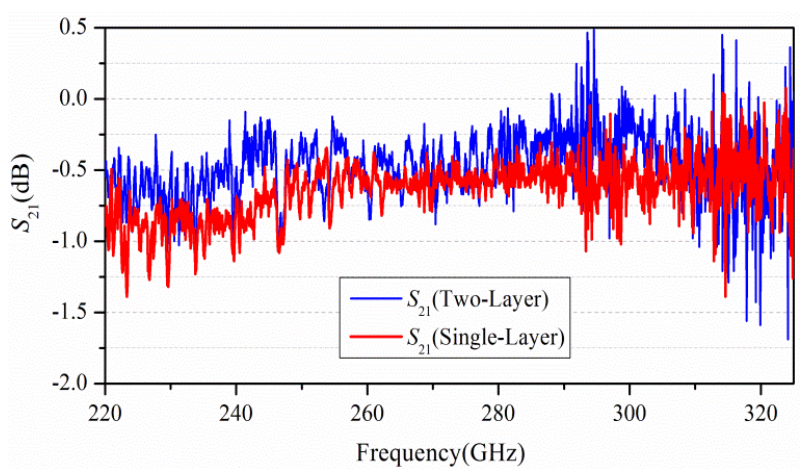

Fig. 3. Measured $S_{21}$ responses of two $14.97 \mathrm{~mm}$ long WR-3 waveguides (From [10]). One was fabricated using single SU8 deposition process labelled as 'Single-layer' and the other using double deposition process labelled as 'Two-layer'.

\section{H. DEVICES}

SU8 has been primarily used in MEMS [6]. In the application described in this paper, this photo-imageable resist is used as the structural material for building 3D coaxial and waveguide devices in a multilayered fashion, as illustrated in Fig. 1 and Fig. 2. The thickness of each layer ranges from 0.1 $\mathrm{mm}$ to about $1 \mathrm{~mm}$. Rectangular coaxial lines have been demonstrated up to the W-band with a cross section of 0.456 $\mathrm{mm}$ by $0.6 \mathrm{~mm}$ [7], [8]. Full-height standard waveguides have been demonstrated up to WR-1.5 band (500-750 GHz) with a cross section of $0.191 \mathrm{~mm}$ by $0.381 \mathrm{~mm}$ [9]-[11]. For the WR-3 waveguides, an attenuation of $0.01-0.05 \mathrm{~dB} / \mathrm{mm}$ across the band has been achieved using the SU8 process as shown in Fig. 3. This is comparable to one of the best machined metal waveguides with an average attenuation of $0.02 \mathrm{~dB} / \mathrm{mm}$ over the same band [10].

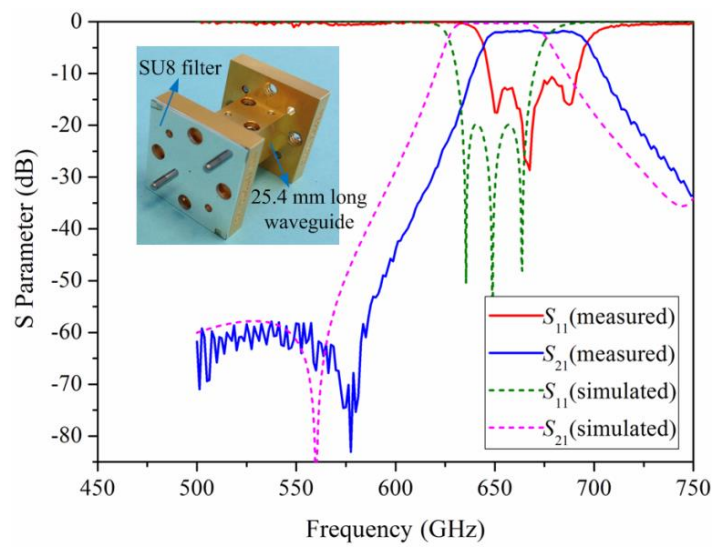

Fig. 4. Performance of the SU8 WR-1.5 waveguide filter. The measurement taken includes the contribution from a $25.4 \mathrm{~mm}$ long metal waveguide which is attached to the SU8 device (inset).

A range of passive devices from $30 \mathrm{GHz}$ to just under 1 $\mathrm{THz}$ have been demonstrated. Various types of wideband or narrow-band filters have been implemented using either rectangular coaxial (up to $110 \mathrm{GHz}$ ) or waveguide (up to 700 $\mathrm{GHz}$ ) structures. These include stub filters [7], interdigital filters, waveguide filters [11] and cavity filters [12]. Fig. 1 shows a W-band stub filter and Fig. 4 shows the response of a WR-1.5 waveguide filter, representing one of the highest operation frequencies demonstrated by a micromachined filter [11]. Directional couplers [5] and Butler matrices [13] have been designed to feed $38 \mathrm{GHz}$ and $63 \mathrm{GHz}$ air-filled patch arrays [14]. Slotted waveguide antennas with various feeding configurations have been fabricated at $300 \mathrm{GHz}$ for gain enhancements and beam scanning [15]. Fig. 2 shows one of these. A common feature of all above-mentioned devices is the air-dielectric (or air-filled) 3D structure which essentially eliminates any dielectric losses.

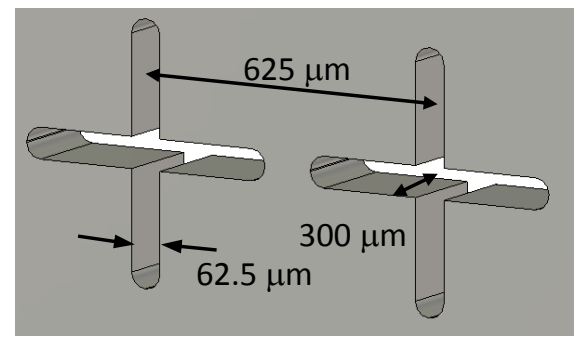

(a)

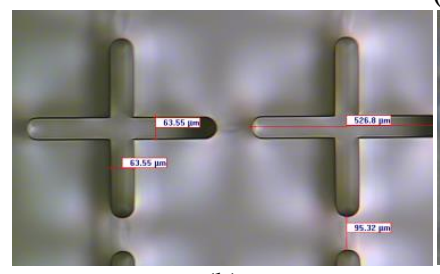

(b)

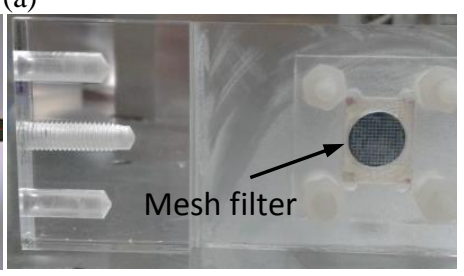

(c)
Fig. 5. Micromachined thick mesh filters. (a) Diagram of the unit cell; (b) Microscopic view of the bare SU8 piece before metallization; (c) Fabricated mesh filter in the sample holder for freespace measurements.

Most recently, the SU8 based lithography process has been adopted to produce freestanding thick mesh filters [16], also knowns as frequency selective surfaces (FSSs), at $300 \mathrm{GHz}$. As shown in Fig. 5, the thickness of the mesh structure is five times of the width of the cross-shaped slots. This large thickness not only increases the mechanical strength but also enhances the resonant quality factor. This is an extra degree of freedom in controlling the transmission characteristics of the FSS. Further shaping of the passband characteristics have been demonstrated using a stacked structure of multiple micromachined mesh filters. This unique low-cost fabrication process is favorably suited for $\mathrm{THz}$ devices for free-space sensing and material characterizations.

\section{FABRICATION AND MEASUREMENTS}

The SU8 fabrication process is based on photolithography with multiple steps of critically controlled baking in order to form stable cross-linked structures. This has been detailed in [17], [18]. The rectangular coaxial and waveguide structures are mostly formed of four or five layers of equal thickness. The simplest process involves a single deposition of SU8 and 
one step UV exposure forming a single layer. An advanced double-deposition process with two exposures has also been successfully demonstrated to produce a single block of double-layered structures in order to reduce the number of contact interfaces when the multiple SU8 layers are stacked together to form the 3D structure.

Measuring theses micromachined devices is a significant challenge due to the non-standard port structures. For the rectangular coaxial devices, as shown in Fig. 1 a transition from the rectangular coax to a suspended thick CPW line is devised to facilitate interconnections with mm-wave on-wafer probes. For the $\mathrm{THz}$ waveguide devices, without a reliable and secure interconnection between the micromachined waveguide and the standard metal flange, the connection loss may well dominate the measured transmission and reflection responses. Therefore, a lot of effort has been made to ensure the reliability and repeatability of the measurements. Extra transition structures [9] or external testing fixtures [19] have been used. The antenna in Fig. 2 contains a matched rightangle bend, designed to realise secure and accurate contact with standard metal waveguide flanges.

\section{CONCLUSIONS}

There have been significant advancements of various micromachining technologies to manufacture $3 \mathrm{D} \mathrm{mm}$-wave and $\mathrm{THz}$ devices with sub-millimeter dimensions. The SU8 based multilayer fabrication technique has demonstrated its potential to be one of the most cost-effective and simple with low facility investment but good capability. This technique has delivered a range of passive devices such as filters, couplers, antennas and arrays. It can be adapted to devices operating over a wide frequency range from $30 \mathrm{GHz}$ up to 1 THz. The fabrication process has been advanced from single deposition to multiple deposition of SU8, resulting in much improved device performance. Different interconnection methods have been experimented and much improved reliability and accuracy in the measurement have been achieved. Although the current fabrication process has achieved competitive performance compared with other micromachining, as well as metal machining techniques, a further reduction of the fabrication tolerance and assembly error is most desirable. One future research direction is to explore the integration of micromachined passives with active components for sensors and communication systems.

\section{REFERENCES}

[1] L. Chen, A. Arsenovic, J. R. Stanec, T. J. Reck, A. W. Lichtenberger, R. M. Weikle, II, and N. Barker, "A micromachined terahertz waveguide 90 twist", IEEE Microw. Wireless Comp. Lett., vol. 21, no. 5, pp. 234-236, 2011.

[2] Z. Popovic, "Micro-coaxial micro-fabricated feeds for phased array antennas", in 2010 IEEE Int. Symp. Phased Array Systems and Technology, Oct. 2010, pp. 1-10.

[3] K. Leong, K. Hennig, C. Zhang, R. Elmadjian, Z. Zhou, B. Gorospe, P. Chang-Chien, V. Radisic, W. Deal, "WR1.5 silicon micromachined waveguide components and active circuit integration methodology", IEEE Trans. Microw. Theo. Tech., vol. 60, no. 4, pp. 998-1005, 2012.

[4] C. E. Collins et al., "A new micro-machined millimeter-wave and terahertz snap-together rectangular waveguide technology," IEEE Microw. Guided Wave Lett., vol. 9, no. 2, pp. 63-65, Feb. 1999.

[5] Y. Wang, M. Ke, M. J. Lancaster, F. Huang, "Micromachined millimeter-wave rectangular-coaxial branch-line couplers with enhanced bandwidth", IEEE Trans. Microwave Theory Tech. . vol. 57, no. 7, pp. 1655-1660, 2009.

[6] H. Lorenz, M. Despont, N. Fahrni, N. Labianca, P. Vettiger, and P. Renaud, "EPON SU-8: a low-cost negative resist for MEMS," in Proceedings of Micro Mechanics Europe, Barcelona, 1996, pp. 32-35.

[7] M. J. Lancaster, J. Zhou, M. Ke, Y. Wang, K. Jiang, "Design and high performance of a micromachined K-band rectangular coaxial cable", IEEE Trans. Microwave Theory Tech.. vol. 55, no. 7, pp. 1548-1553, 2007.

[8] M. Ke, Y. Wang, M. J. Lancaster, "Micromachined rectangular coaxial line and cavity resonator for $77 \mathrm{GHz}$ applications using SU8 photoresist", in Asia Pacific Microwave Conference, Hong Kong, 2008.

[9] X. Shang, M. Ke, Y. Wang, M. Lancaster, "Micromachined Wband through waveguide and filter with two H-plane back-toback bends", IET Microw. Antenn. Propag., vol. 5, no. 3, pp. 334-339, 2011.

[10] X. Shang, M.L. Ke, Y. Wang, M.J. Lancaster, "WR-3 band waveguides and filters fabricated using SU8 photoresist micromachining technology", IEEE Trans. Terahertz Sci. Tech., vol. 2, no. 6, pp. 629-637, Nov 2012.

[11] X. Shang, Y. Tian, M.J. Lancaster, S. Singh, "A SU8 micromachined WR-1.5 band waveguide filter", IEEE Microw. Wireless Comp. Lett., vol. 23, no. 6, 300-302, 2013.

[12] Y. Wang, M. Ke, M. J. Lancaster, "Micromachined $38 \mathrm{GHz}$ cavity resonator and filter with rectangular-coaxial feed-lines", IET Microw. Anten. Propag., vol. 3, no. 1, pp. 125-129, Feb. 2009.

[13] Y. Wang, M. Ke, M. J. Lancaster, "A Ka-band butler matrix with antenna array based on micromachined rectangular coaxial structures", in 39th European Microwave Conference, Sept. 2009, pp. 739-742.

[14] Yi Wang, M. Ke, M. J. Lancaster, "Micromachined air-filled patch antennas for millimetre-wave applications", Microw. Opt. Tech. Lett., vol. 52, no.6, 2010, pp. 1345-1347.

[15] Y. Wang, M. Ke, M. Lancaster, J. Chen, "Micromachined 300 $\mathrm{GHz}$ SU-8-based slotted waveguide antenna", IEEE Anten. Wireless Propag. Lett., vol. 10, pp. 573-576, 2011.

[16] Yi Wang, B. Yang, Y. Tian, R, S. Donnan, M. J. Lancaste, "Micromachined thick mesh filters for millimeter-wave and terahertz applications", IEEE Trans. Terahertz Sci. Tech., vol. 4, no. 2, pp. 247-253, Mar. 2014.

[17] M. Ke, Y. Wang, S. Wei, K, Jiang, M. Lancaster, "Precision microfabrication of milli-metre wave components", in 9th International Conference and Exhibition on Laser metrology, machine tool, CMM and robotic performance (LAMDAMAP), June 2009.

[18] Y. Tian, X. Shang, M.J. Lancaster, "Fabrication of multilayered SU8 structure for terahertz waveguide with ultralow transmission loss", J. Micro/Nanolithography, MEMS, and MOEMS, vol. 13, no. 1, 013002, 2014.

[19] X. Shang, M. J. Lancaster, M. Ke, Y. Wang, "Measurement of micromachined submillimeter waveguide circuits", ARFTG 76th Microwave Measurement Symposium, Clearwater Beach, FL. November 30th - December 3rd 2010. 